\title{
Synthesis and Characterization of Composite Membranes Based on Carbon Functionalized with Gold Nanoparticles
}

\author{
ALEXANDRA GEORGIANA VATUI (MOISE) ${ }^{1,2}$, SORINA NICOLETA VALSAN ${ }^{1 *}$, CORNELIU FRATILA ${ }^{1}$, ANDREEA NICO LETA GHITA ${ }^{1,2}$, \\ ARCADII SOBETKII ${ }^{1}$, DANIEL CRISTIAN MIHAIESCU ${ }^{1}$, ANCA ELENA SLOBOZEANU ${ }^{1,2}$, ROXANA TRUSCA ${ }^{3}$ \\ ${ }^{1}$ National Research \& Development Institute for Non-Ferrous and Rare Metals (INCDMNR-IMNR), 102 Biruintei Blvd., 077145, \\ Pantelimon, Romania \\ ${ }^{23}$ Politehnica University of Bucharest, Faculty of Materials Science and Engineering, 313 Splaiul Independentei, 060042, Bucharest, \\ Romania \\ ${ }^{3}$ Politehnica University of Bucharest, 313 Splaiul Independentei, 060042, Bucharest, Romania
}

The purpose of this experimental laboratory work is to obtain composite membranes having carbon and gold nanoparticles in the matrix. Consideration was made of using graphene oxide, a material which, under certain conditions, can generate membranes by a self-assembly process under vacuum in an aqueous or organic medium. The cellulose acetate (CA) - graphene oxide (GO) composite membranes have been functionalized with gold nanoparticles (AuNP) by two technological processes: thermal evaporation and ultrasonic mixing of stable graphene oxide and gold suspensions. The stable suspensions containing gold nanoparticles were obtained by chemical reduction of aqueous solutions of Tetrachloroauric acid trihydrate $(\mathrm{HAuCl})$ precursors with sodium citrate under high temperature conditions. Dispersibility of carbon nanostructures and precious metal nanoparticles has been studied with a DLS granulometer - Malvern Instruments Zetasizer ZS90. It was determined the mean particle size and Zeta potential function of the suspensions $\mathrm{pH}$. The graphite based membranes were further subjected to various characterizations techniques. It was found that both the surfaces and the cross-sections were functionalized with gold particles. The presence of Au particles was revealed both on the membrane surface and also inside its structure. Hydrophilic tests demonstrate that the membrane filters have potential to be used in various applications.

Keywords: filtering composite membranes, carbon, graphene, graphene oxide, gold nanoparticles

Nanotechnology has emerged as an alternative tool for the development of new-grade products to meet the increasing demand of industrial sectors for advanced functional materials. In particular, nanostructured materials, such as carbon nanotubes and graphene, have been developed as modeling systems in nanoscience and nanotechnology [1,2]. These carbon-based materials are a special class and have attracted great attention because of their superior surface area and their exceptional electronic, mechanical, thermal and adsorbtion properties $[3,4]$. Graphene and graphene oxide are undoubtedly the materials of the future. Graphene is a two-dimensional material composed of a $\mathrm{sp}^{2}$-hybridized hexane carbon network, while graphene oxide (GO) is a chemically modified graphene with hydroxyl, carboxyl and epoxy functional groups. This form of functional graphene is capable of producing dispersions in water consisting essentially of single-layer $\mathrm{GO}$ sheets [5]. This material was used as a promising construction block for the preparation of new composites [6].

Recently, graphene oxide is very intensively studied in composite filter membranes that can combat the biofouling phenomenon. A very interesting combination of graphene oxide and nanoparticles of precious metals ( $\mathrm{Au}$, $\mathrm{Ag}, \mathrm{Pd}$, etc.) is increasingly being studied in the micro and nanofiltration field. Consequently, precious metal nanoparticles assembled on graphene oxide sheets (GO$\mathrm{Ag}, \mathrm{Au}$ etc.) were used as new antibacterial systems [79]. The large surface area of $G O$ sheets serves as a support for the growth and stabilization of nanoparticles, and prevents them from forming aggregates [10].

Moreover, these nanocomposites based on $\mathrm{Au}$ and $\mathrm{Ag}$ have excellent antimicrobial properties and represent a viable alternative to the classical processes used at this time. Once in contact with the cell membrane of the microorganisms, graphene and graphene oxide create an impermeable film that makes normal exchange between cellular and extra cellular media impossible, leading to cell death. Gold nanoparticles deposited on the porous surface of graphene oxide form non-toxic catalytic systems can catalyze highly selective by low temperature reactions such as partial oxidation [11]. Also, gold nanoparticles can be used to purify water by several mechanisms [12]: (1) removal of contaminants by physical adsorption or chemical reaction; (2) contaminant degradation by catalytic reaction and (3) colorimetric sensory detection of pollutants.

\section{Experimental part}

Composite membranes of cellulose acetate - graphene oxide were functionalised with Au nanoparticles by two different methods:

- Thermal evaporation, the method which produces a golden layer on the surface of the membrane and allows control over the thickness and permeability of the resulted layer. Generally, for applications in the filtering membranes, layers of about 20-30 nm thicknesses are deposited.

- Stable aqueous suspensions based on graphene oxide and gold nanoparticles. In this way, the composite membranes can be decorated with gold nanoparticles both on the surface and inside the structure.

\section{Preparation of stable suspensions for vacuum filtration on cellulose acetate membranes \\ In order to filter under vacuum on commercial membrane of cellulose acetate with a diameter of $47 \mathrm{~mm}$ and a 0.2 microns porosity, it was used an aqueous dispersion of commercial graphene oxide with a $4 \mathrm{mg} / \mathrm{mL}$}


concentration. The initial solution of graphene oxide is a concentrated solution that cannotbe used as such because it generates large thicknesses (tens of microns) that are not very permeable and allow passing through them a low flow of liquid. It is known that the flow of liquid through a membrane is inversely proportional to its thickness. Also, if the thickness of the deposited layer is too high, the membrane can detach from the support, so it is necessary to work with dilute graphene oxide solutions to generate stable suspensions over time, with increased effectiveness in composite membranes. It was experimentally established that a $0.25 \mathrm{mg} / \mathrm{mL}$ concentration allowed to obtain a well-dispersed solution, which corresponds to a permeable membrane having a good adherence to the support. After the ultrasonic and centrifugation treatment, the suspensions were analysed in order to check the dimensional stability. This influences the vacuum filtration process, favouring self-assembly of the membranes on the cellulose acetate support.

\section{Analyzing the stability of obtained suspensions}

Analysis of particle size distribution by DLS method

The diluted graphene suspension was stable, the stage preceding DLS (Dynamic Light Scattering) analysis was ultra-sonication for $15 \mathrm{~min}$. The results revealed an average particle size of $453 \mathrm{~nm}$ (fig. 1 - left). The Polydispersity Index (0.47) highlighted a moderate monodisperse suspension. The DLS analysis takes into account the adsorbed layer on the surface of the nanoparticle, a hydrated/solvated surface. Therefore, the average particle size measured by the DLS method is greater than the average size of the synthesized nanoparticles and measured by SEM/TEM.
Zeta potential analysis

Zeta potential indicates electrophoretic mobility combined with particle velocity measurement. The value of zeta potential provides an indication of the stability of the colloidal system. It is considered that the interval between $-30 \mathrm{mV}$ and $+30 \mathrm{mV}$ shows an unstable system, and values greater than $+30 \mathrm{mV}$ and below $-30 \mathrm{mV}$ are the indication of colloidal suspension stability. The most important factor affecting zeta potential is the $\mathrm{pH}$. The results indicated an optimal suspension for measurements. The value of zeta potential places the suspension in stable systems domain (fig. 1 - right).

\section{Composite membranes obtained by vacuum filtration}

The graphene oxide plates randomly overlap during vacuum filtration after a given direction, but without strict rules or transport schemes. They form a layered structure of parallel planes with different densities and thicknesses. It has been found that water adheres to GO plates, hydrogen atoms bind to carbon atoms. The result was a thin film of flexible graphene oxide. By adjusting the graphene oxide content in water, the thickness of the film change.

Figure 2 shows the vacuum filtration system for obtaining composite membranes. The system is connected to a membrane vacuum pump.

Ithas been found that the physico-mechanical properties of macroscopic membranes are closely related to the dispersion concentration and, implicitly, to their filtration rate. Using very dilute solutions can obtain thin films with thicknesses between 1 and 2 microns and high elasticity. There is a correlation between the concentration of

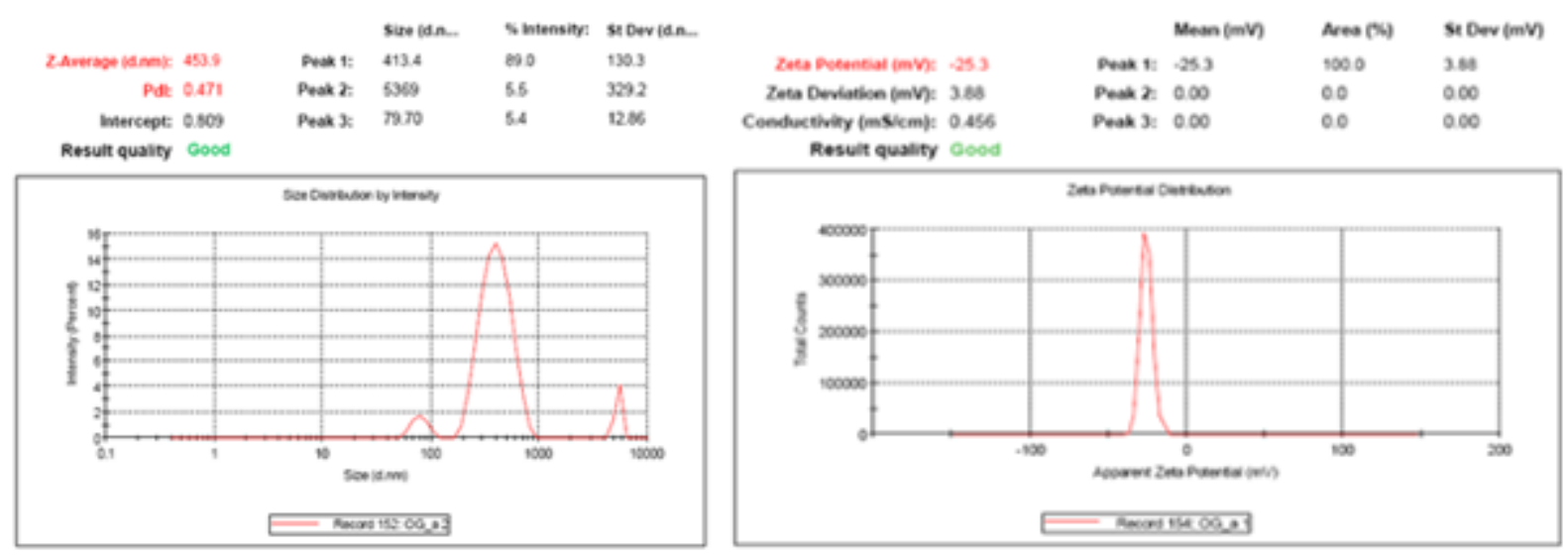

Fig. 1. DLS analysis (left) and Zeta potential of graphene oxide (right)

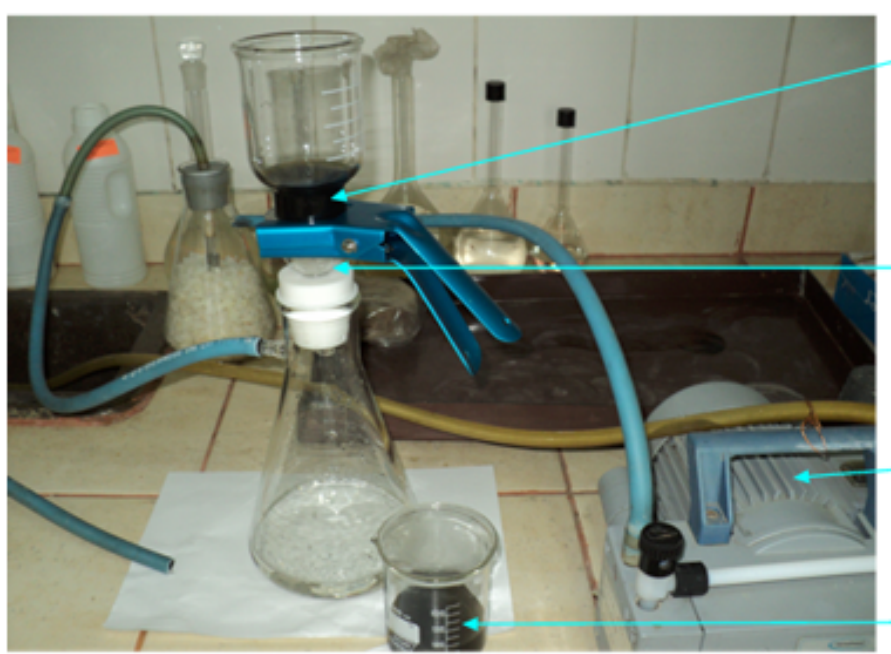

$300 \mathrm{ml}$ graduated flask With graphene solution

Pyrex frit filter with

$10 \mu \mathrm{m}$ porosity and 47 $\mathrm{mm}$ cellulose acetate membrane

Fig. 2. Vacuum filtration system (in order to achieve composite membranes on the basis of the graphene oxide)

Diaphragm vacuum pump ( 9 mbar) 
solutions and the amount of solution used to filter according to the intended purpose. Atthe end of the filtration operation (approx. 4 hours), flexible and compact graphene oxide membranes are obtained, as can be seen in SEM (scanning electron microscopy) analysis (fig. 3 - left). In figure 3 right side there are two composite membranes obtained from solutions with different concentrations. The colour of the membranes varies depending on the concentration of the suspension used in the process, with the increase in colour changing from brown, to dark brown, to black (fig. 3 - right).
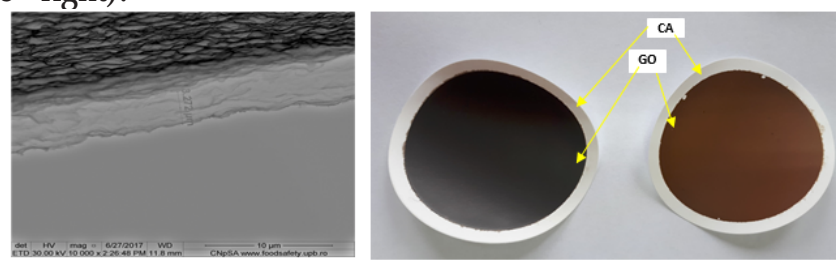

Fig. 3. SEM micrograph of GO-based composite membranes deposited from stable dispersions under vacuum (left) and membranes obtained after vacuum filtration (right)

Membranes functionalisation with gold nanoparticles by thermal evaporation method

This method involves physical processes such as heating the material in vacuum with an electrical resistance (J oule effect), until a high vapour pressure is created that allows the diffusion of the material and the condensation deposition on the cold workpiece. A very high vacuum is required so that the atoms or molecules from the thermally evaporated source reach the substrate without collisions with the residual gas molecules in the deposition chamber. The physical properties, the crystalline structure, the composition and the degree of purity of the layer depend on the pressure of the gases in the evaporation chamber, the traces of oxygen, nitrogen, etc.

Materials used for gold nanoparticles thermal deposition

As substrate to be coated, cellulose acetate filters with graphene oxide were used. The raw material used for deposition consisted of metallic gold with the following characteristics: purity $99.99 \%$, density $19.32 \mathrm{~g} / \mathrm{cm}^{3}$, melting point: $1062^{\circ} \mathrm{C}$, vapour pressure temperature $10 \mathrm{e}^{-6} \mathrm{~T} 947^{\circ}$ C, acoustic impedance: $23.17 \mathrm{~g} / \mathrm{cm}^{2} \mathrm{~s}$. The equipment used for gold deposition experiments were ultrasonic bath for substrate preparation and thermal evaporation equipment - TORR THE5-750W (fig. 4 - left). Functional membranes of type $A C / G O /(\mathrm{Au}$ ) (fig. 4 - center) were obtained and these were analysed by SEM (fig. 4 - right).

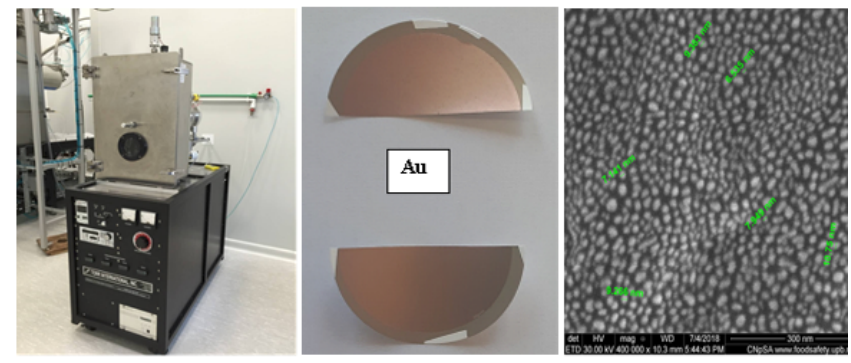

Fig. 4. PVD thermal evaporation installation (left); CA / GO / Au membranes (center); SEM micrograph of the membrane surface coated with Au nanoparticles (right)

As it can be observed, there is a uniform distribution of gold nanoparticles of approximate size between 6-10 nm across the surface. Nanoparticles are spherical and do not form a continuous waterproof layer for liquids. In this case, a controlled porosity of the membranes that can become selective and only some small ions can pass through the created spaces.
Membrane functionalisation from stable aqueous suspensions based on graphene oxide and gold nanoparticles

Experimental laboratory work were conducted in two main steps: obtaining independent stable aqueous suspensions based on Au nanoparticles and graphene oxide; obtaining of GO / Au composite membranes.

In the first step, from precursor solutions of $\mathrm{HAuCl}_{4}$ (Acros Organics - Tetrachloroauric acid trihydrate) by reduction with sodium citrate stable aqueous was obtained suspension of gold nanoparticles (fig. 5- left). Next, stable aqueous suspensions of graphene oxide with a concentration of $0.25 \mathrm{mg} / \mathrm{mL}$ were obtained (fig. 5-center and right). The two types of suspension are mixed and homogenized by ultrasonography.

The GO / Au membrane was obtained by vacuum filtration (100 mbar) in an imposed direction of the composite suspension, using a cellulose acetate membrane matrix with a porosity of $0.2 \mu \mathrm{m}$. The membrane process involves several steps as follows: - the proper filtration that makes the composite membrane through random combinations made between graphene oxide nanoparticles and gold nanoparticles, specific only to these materials; washing and, implicitly, cleaning the membranes on the filter support in several stages; drying and strengthening the membranes under vacuum for a few hours (2-3 h). In general, in order to be able to use such a membrane for applications it is necessary to dry and consolidate it for up to $20 \mathrm{~h}$ under vacuum.
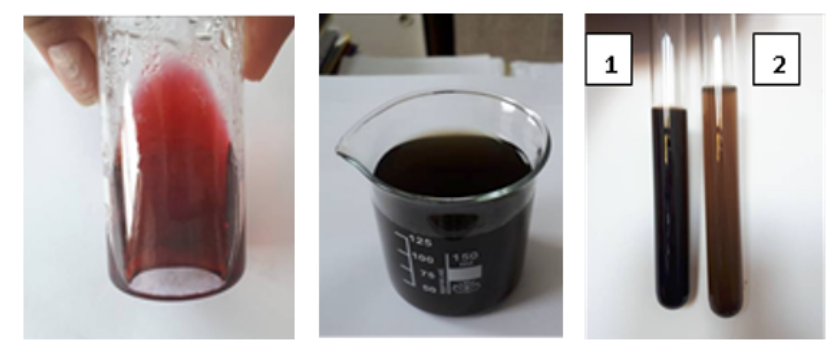

Fig. 5. Suspension with gold - specific colour for gold

nanoparticles (left); Aqueous dispersion of graphene oxide (center): 1 before centrifugation and 2 after centrifugation (right)

Dispersibility of carbon and gold nanostructures in aqueous suspension has been studied with Malvern Instruments Zetasizer ZS90 (fig. 6-left). The mean particle size and Zeta potential of suspensions were evaluated (fig. 6 - right).

The results revealed an average particle size of 7.263 $\mathrm{nm}$. The polydispersity index (0.713) indicated a polydispersed suspension, with a large particle size distribution. This is also noticeable after the configuration of the peaks on the graph (large and flattened) and the statistical ratio of dimensions by intensity.

Zeta potential values based on the $\mathrm{pH}$ of the dispersion indicate stable suspensions.

The two types of suspension are ultrasonic mixed and homogenized. The obtained composite suspension is filtered under vacuum in an imposed direction over a support made of a cellulose acetate membrane with 0.2 $\mu \mathrm{m}$ porosity. The support is designed to provide the GO membrane with the necessary mechanical strength and stability to prevent damage to subsequent filtering processes. The membranes obtained are flexible with gold content on both surfaces (fig. 7 - the left). The surface morphology is shown in figure 7 - center and right and the aspect at the cross-sectioned structure is presented in the micrograph in figure 8. 

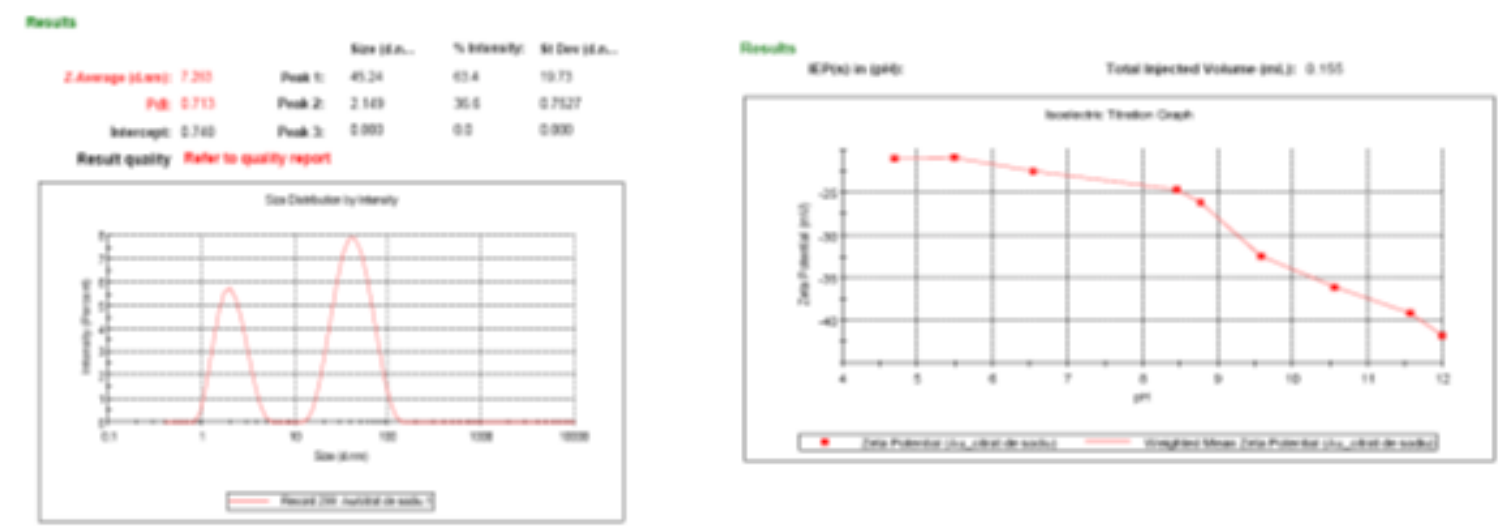

Fig. 6. DLS analysis of the Au nanoparticles suspension (left); Zeta potential of the same suspension (right)
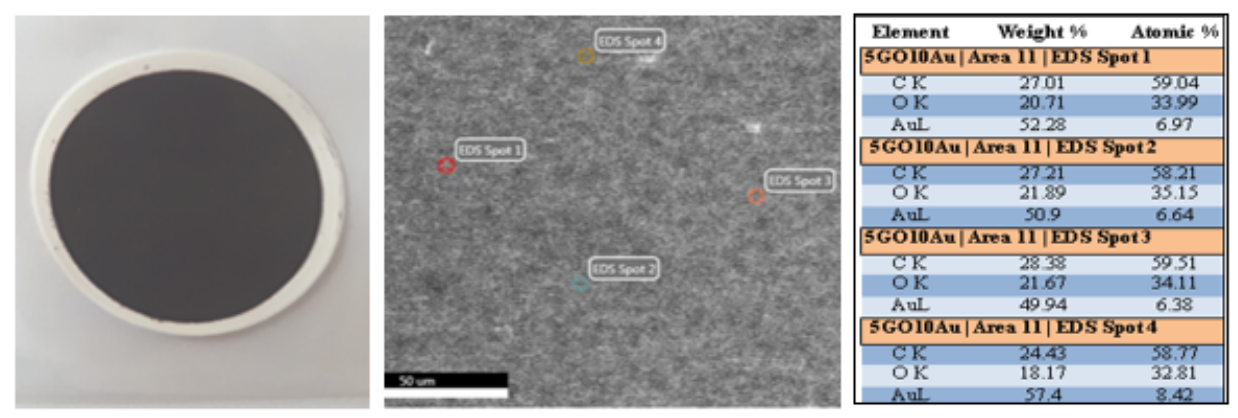

Fig. 7. GO-Au membrane (left); The surface of the GO membrane decorated with gold particles (Spot $1 \div 4$ ) (center); Quantitative analysis of the membrane surface (right)

The presence of gold in the cross-section structure of the GO layer (Spot 1) was observed. The other points belong to the cellulose acetate support and the carbon grid. It is possible to find gold on the surface of the cellulose acetate membrane section.

The obtained membranes were also investigated using a scanning electron microscope with an energy dispersive $X$-ray spectrometer (SEM/EDX). Both surfaces of the composite membranes (Spot $1 \div 4$ ) and their crosssections (Spot 5) were analysed (fig. 9). It was found that both the surfaces and the sections were decorated with gold particles both as nanoparticles and as micronic agglomerates. The gold particles are evenly distributed over the membrane surface. In this case, we are dealing with a fibrous surface consisting of a carbon-gold filament fabric, a uniform compact and adherent to the support. It is thus demonstrated that through this technology composite membranes can be decorated with gold particles on both the surface and inside the membrane structure.

\section{Hydrophilicity test at T- 0 and T-5min moments}

The evaluation of the hydrophilicity of the material consisted at contact angle measurements in the presence of different liquids. That should be a predictive indicator of filtering and separation capabilities of the membrane based on graphene oxide and carbon nanotubes. The surface is moderately hydrophilic, the contact angle decreasing in 5 min from $73.1^{\circ}$ to $33.2^{\circ}$ (fig. 10).

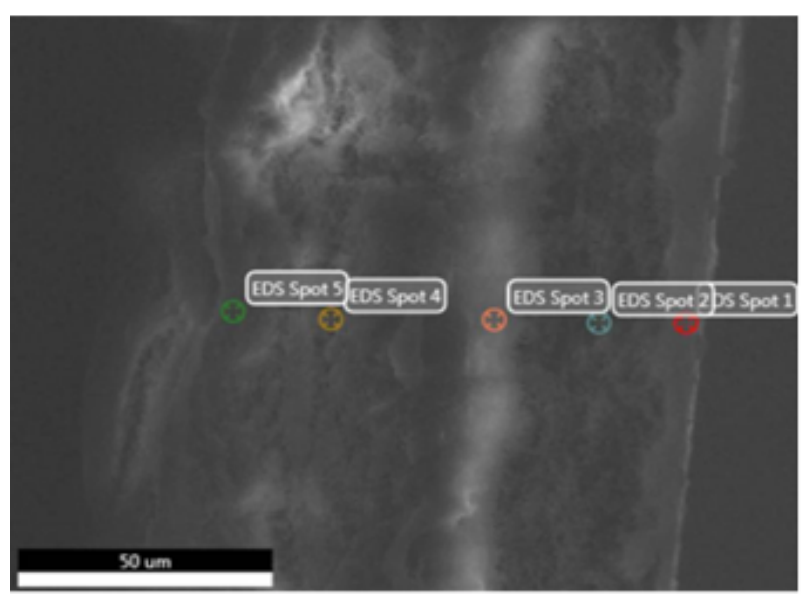

\begin{tabular}{|c|c|c|}
\hline Element & Weight $\%$ & Atomic $\%$ \\
\hline \multicolumn{3}{|c|}{\begin{tabular}{l|l|l|l|l|l}
5 SGOl0Au & Area & EDS Spot
\end{tabular}} \\
\hline $\mathrm{CK}$ & 31.52 & 57.85 \\
\hline $\mathrm{OK}$ & 27.24 & 37.53 \\
\hline $\mathrm{AuL}$ & $\quad 41.24$ & 4.62 \\
\hline \multicolumn{3}{|c|}{$\begin{array}{l}5 \mathrm{SO} 010 \mathrm{Au} \mid \text { Area } 9 \\
\text { EDS Spot } 2\end{array}$} \\
\hline $\mathrm{CK}$ & 48.97 & 56.1 \\
\hline $\mathrm{OK}$ & $\quad 51.03$ & 43.9 \\
\hline \multicolumn{3}{|c|}{ 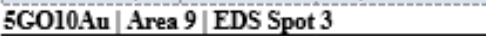 } \\
\hline $\mathrm{CK}$ & $\begin{array}{l}45.11 \\
4\end{array}$ & 52.27. \\
\hline $\mathrm{OK}$ & 54.89 & 47.74 \\
\hline \multicolumn{3}{|c|}{\begin{tabular}{|l|l|l|l|}
5 GO10Au & Area & EDS Spot 4
\end{tabular}} \\
\hline $\mathrm{CK}$ & 43.38 & 50.51 \\
\hline OK & $\quad 56.62$ & 49.49 \\
\hline $5 \mathrm{GOO} 0 \mathrm{Au}$ & \begin{tabular}{|l|l|} 
Area 9 & EDS Spo
\end{tabular} & \\
\hline $\mathrm{CK}$ & 55.54 & 62.46 \\
\hline $\mathrm{OK}$ & 44.46 & 37.54 \\
\hline
\end{tabular}

Fig. 8. Composite CA-GO-Au cross-section membrane (Spot $1 \div 5$ ) (left); Quantitative analysis of the cross section (right) 


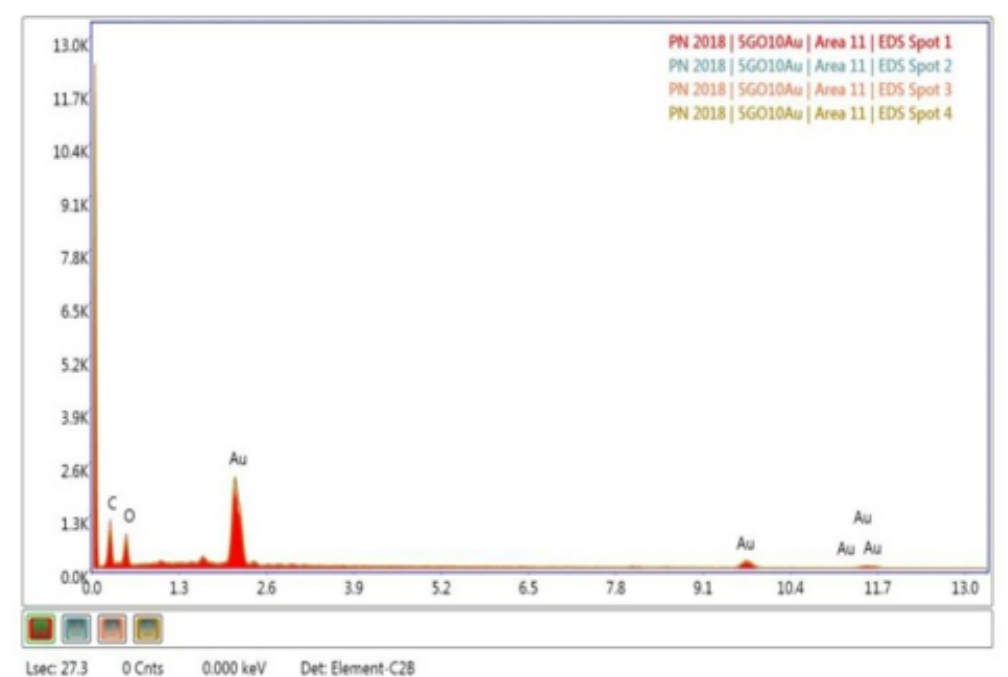

sec 27.3 OCAnts $0.000 \mathrm{keV}$ Det Element-C25
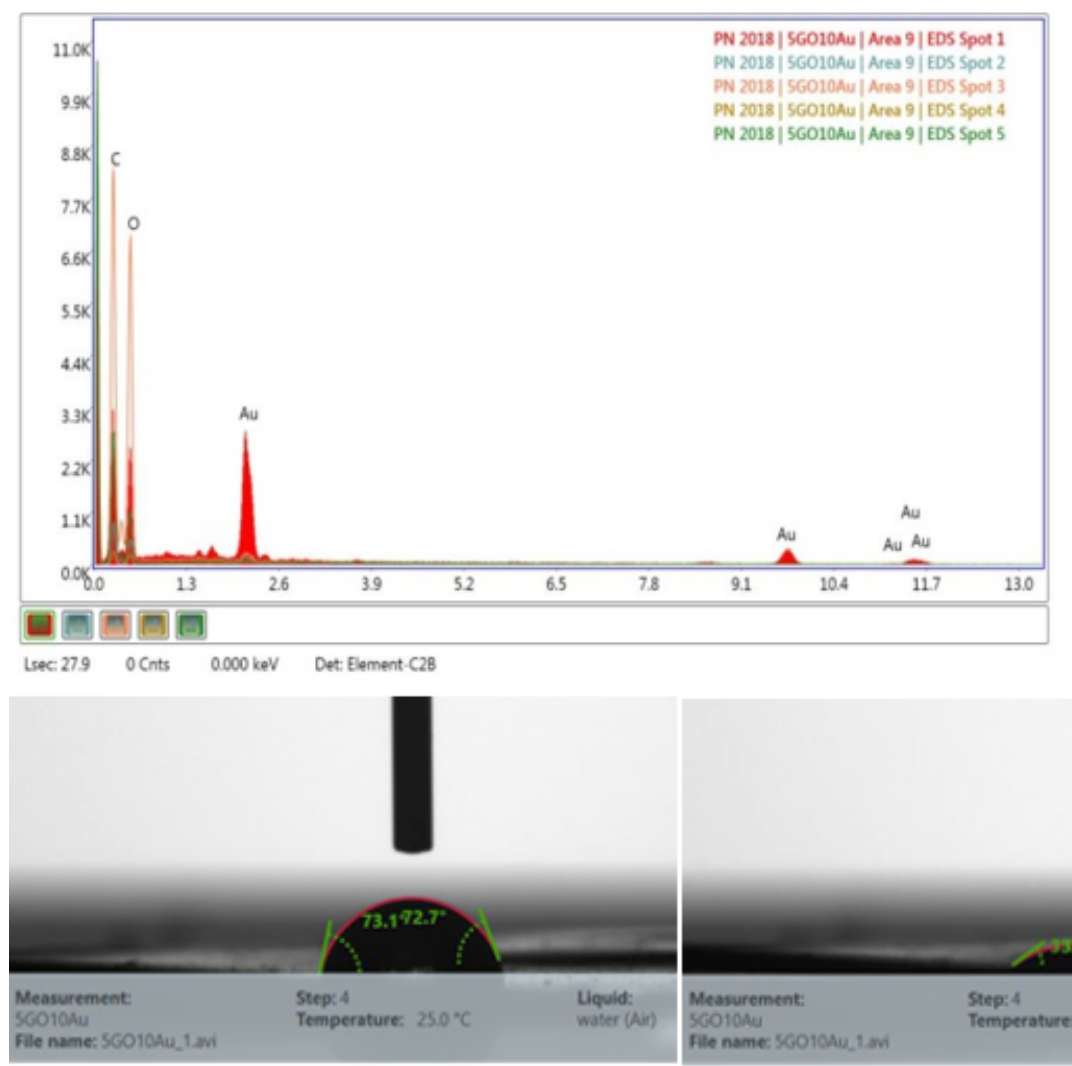

(20)

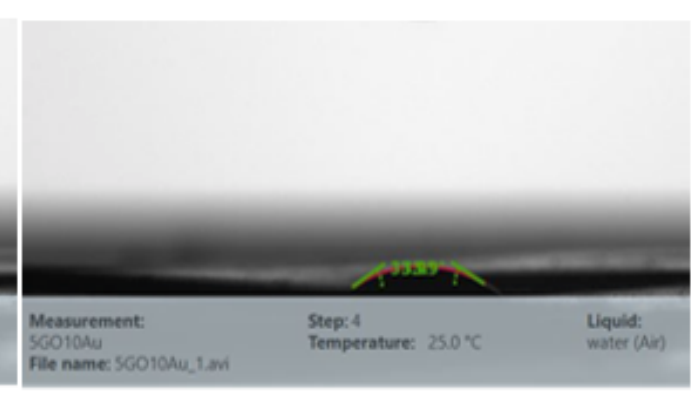

Fig. 10. Water drop at time T-0 min (left); Water drop at time $\mathrm{T}-5 \mathrm{~min}$ (right)
Fig. 9. EDX spectrum: - on the surface of the composite membrane CA-GO-Au (Spot $1 \div 4$ ) (up);

- on the cross-section CA-GO-Au composite membrane (Spot $1 \div 5$ ) (down)

\section{Results and discussions}

The membrane surfaces and their cross-sections were analyzed using SEM/EDX techniques. It has been found that both the surfaces and the cross-section had been impregnated with gold nanoparticles in form of micronized agglomerates. Gold nanoparticles appear on the surface of the layers of graphene oxide and as a spacing element that can alter the size of the filtration channels.

Evaluation of the hydrophilicity of the material, performed by contact angle measurement in the presence of differentliquids, should be a predictive indicator of filtering and membrane separation capabilities based on graphene oxide and carbon nanotubes.

\section{Conclusions}

The reported experimental works have been focused on preparing a composite membranes based on carbon nanomaterials and metal nanoparticles starting from stable aqueous suspensions (CA - GO - Au). It has been shown that graphene oxide $(\mathrm{GO})$ are decorated with $\mathrm{Au}$ nanoparticles on the surface and possibly inside the structure.

Hydrophilicity tests were performed by measuring the contact angle of drops of water on the surface of the GO and CNT membranes. In all cases, contact angles of less than $90^{\circ}$ were observed after 5 min, indicating moderate hydrophilicity of the membranes obtained.

Thus, antimicrobial and antifouling nanofiltration systems with potential applications in advanced water purification can be developed.

Acknowledgement: This work was supported by a grant from the Ministry of Research and Innovation, Program Nucleu, contract no. 23N/2018 (Project code PN 18070301 / 2018).

\section{References}

1. YANG M., HOU Y., KOTOV N.A. - Graphene-based multilayers: Critical evaluation of materials assembly techniques, Elsevier Nano Today 7 (2012) 430-447

2. GEIM A. K. - Random walk to graphene, Nobel Lecture: Rev. Mod. Phys. 83 (2011) 851 
3. WANG G., YANG J ., PARK J., GOU X., WANG B., LIU H. and YAO J . Facile Synthesis and Characterization of Graphene Nanosheets, J. Phys. Chem. C vol. 112, no. 22 (2011) 8192-8195

4. BOGDAN D., RIZEA G.A., ION I., ION A.C. - Ammonium Adsorption on Exfoliated Graphite Nanoplatelets, Rev. Chim. (Bucharest) 69, no.10, 2018, p. 2625- 2632

5. PARVEZ K. - Chemical exfoliation of graphene and its application in organic electronics and energy storage devices Dissertation-zur Erlangung des Grades Doktor der Naturwissenschaften im Fachbereich Chemie, Pharmazie, und Geowissenschaften der J ohannes GutenbergUniversität Mainz und in Kooperation mit dem Max-Planck-Institut für Polymerforschung Mainz vorgelegt von Khaled Parvez geboren in Gazipur/Bangladesh Mainz, 2014

6. MIAO C., ZHENG C., LIANG O., XIE Y.-H. -(Book) Chemical Vapor Deposition of Graphene, Physics and Applications of Graphene Experiments (2011) Dr. Sergey Mikhailov (Ed.), ISBN: 978-953-307-2173
7. SIMOES M., SIMOES L., VIEIRA M.J . - A review of currentand emergent biofilm control strategies, Food Sci. Technol. 43 (2010) 573-583

8. ROMERO D., KOLTER R. - Will biofilm disassembly agents make it to market? Cell 19 (2011) 304-306

9. HOUDT R.V., MICHIELS C.W. - Biofilm formation and the food industry, a focus on the bacterial outer surface, J. Appl. Microbiol. 109 (2010) 1117-1131

10. NAJ AFABADI A.T., GYENGE E. - High-yield graphene production by electrochemical exfoliation of graphite: Novel ionic liquid (IL)acetonitrile electrolyte with low IL content, Elsevier Carbon 71 (2014) 58-69

11. HE Y., ZHANG N., GONG Q., LI Z., GAO J., QIU H. - Metal nanoparticles supported graphene oxide 3D porous monoliths and their excellent catalytic activity, Materials Chemistry and Physics 134 (2012) $585 \mathrm{e} 589$

12. QIAN H., PRETZER L.A., VELAZQUEZ J.C., ZHAO Z. and WONG M.S., Gold nanoparticles for cleaning contaminated water, J Chem Technol Biotechnol 88 (2013) 735-741

$\overline{\text { Manuscript received: } 6.12 .2018}$ 